\title{
La traduction espagnole des pronoms clitiques français en et $y$
}

Kris Buyse

Katholieke Universiteit Leuven (kris.buyse@arts.kuleuven.ac.be)

Handelshogeschool Antwerpen (kris.buyse@hha.be)

\section{Introduction}

Une première étude, purement descriptive, de la traduction espagnole des clitiques en et $y$, qui n'ont pas d'équivalent en espagnol, donne l'impression qu'on les traduit de façon assez arbitraire. Ceci peut être la raison pour laquelle le petit nombre d'études qui y consacrent quelques lignes, n'arrivent pas à présenter des stratégies de traduction détaillées et nuancées. Dans cet article, j’essaierai de démontrer comment on peut pallier les problèmes qui émanent de l'état de la question des études sur la traduction de en et y (1), en se basant sur un corpus électronique bilingue français-espagnol (2) qui permet d'abord de classifier tous les emplois de en et y par ordre de fréquence (3), ensuite de comparer la traduction de en et y avec celle des autres clitiques objet (4), pour induire enfin, à partir d'une analyse linguistique de la traduction de en et y proprement dite (5), un système de traduction global (6) et des stratégies de traduction spécifiques pour chacun des emplois de enet $\mathrm{y}(7)$.

\section{Etat de la question}

Un état de la question du peu d'études qui consacrent quelques lignes à la traduction espagnole de en et $y$, permet de dégager les critiques suivantes.

a) Ces études ne décrivent pas tous les emplois de en et y (p.ex. on ne traite pas le 'en culminatif de Blanche-Benveniste (1984: 79) (1), ni le en ou y complément d'adjectif (2).

$$
\begin{aligned}
& \text { Il en perd la tête } \\
& \text { Il y est sensible }
\end{aligned}
$$

$\beta)$ Les auteurs proposent des stratégies contradictoires pour les mêmes emplois (p.ex. le $y$ non-locatif se traduit selon Coste \& Redondo (1965) par un syntagme prepositionnel (PP) et selon Criado de Val (1958) par un implicite; d'autre part, le $y$ locatif se traduit selon Coste \& Redondo (1965) par un adverbe locatif, précédé ou non par une preposition, et selon Badía Margarit (1947) par un implicite.

$\gamma)$ En et $y$ sont généralement présentés comme des exceptions parmi les autres clitiques français: ces derniers se traduiraient toujours par leurs équivalents clitiques espagnols, ce qui les rendrait totalement différents de en et $y$. Il n'y aurait donc pas de liens entre la traduction de en et $y$ et celle des autres clitiques objet. Mais on ne donne pas de preuves de cela, p.ex. sous forme d'indexes de fréquence. 


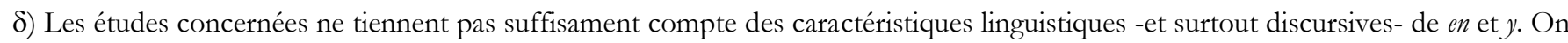
propose p.ex. de les traduire par des PP, sans rendre compte des différences phonétiques et discursives entre un clitique et un PP:

"J'en rêve souvent, de ça" se traduit par un Pprep. (...) En espagnol, il n'existe pas de forme clitique dans ce cas (...); toutes les constructions d'un P3 [un en adverbial, comme dans Il en doute, k.b.] se rangent dans le paradigme P4 [un PP sans proportionnalité clitique, k.b.]. (Deleye, 1988, 54))

La différence discursive entre un groupe prépositionnel et un clitique est pourtant très grande. Dans la hiérarchie référentielle de l'espagnol proposée par Fant (1984: 90), le niveau des PP est deux fois plus haut que celui des clitiques:

$$
S N(+P P)>\text { pr. tónico }>\text { pr. clítico/desinencia verbal }>\phi i
$$

On peut se demander si cette différence n'a pas d'influence sur la traduction de ces clitiques.

ع) Finalement, la représentativité de ces stratégies doit être mise en question, puisqu'elles ne sont pas basées sur une étude variationniste d'un ou de plusieurs corpus parallèles contextualisés. Les traductions proposées ne sont pas accompagnées d'un index de fréquence.

\section{Elaboration d'un corpus électronique bilingue français-espagnol}

C'est pourquoi j'ai commencé par constituer un corpus suffisament homogène et en même temps hétérogène, afin de pouvoir étudier, en premier lieu, tous les emplois et les caractéristiques de en et $y$ et des autres clitiques français, et, en second lieu, leurs traductions. Le corpus comporte trois parties, dont la première est entièrement informatisée. C'est de cette partie que sont induites des stratégies de traduction. Celles-ci ont été confrontées aux exemples des deux autres parties, qui diffèrent de la première en termes de grandeur, de format et de genre. La première partie contient 113 textes journalistiques du Monde Diplomatique, publiés entre '92 et '94 et traduits par 16 traducteurs. La deuxième partie comprend des essays de Roger Garaudy, traduits en espagnol. Et la dernière partie contient des traductions de romans de Ben Jelloun, Pérec, Oursel et Yourcenar. Le corpus entier contient 2990 cas de $e n$ et $y$ traduits par 20 traducteurs hispanophones différents, masculins et féminins. 1122 ocurrences sont locutionnelles, et donc improductives, comme $y$ dans il $y$ a.

\section{Classification de tous les emplois de en et y par ordre de fréquence}

Ce corpus a servi d'abord pour établir la liste complète des emplois de en et $y$ et de les ordonner par ordre de fréquence, ce qui donne le résultat suivant:

\begin{tabular}{|c|c|c|}
\hline$E N$ & & \\
\hline En adverbial $(38 \%)$ & Il s'en souvient & Lo recuerda \\
\hline $\begin{array}{l}\text { En de construction secondaire }(31 \%) \\
\text { en complément d'adjectif } \\
\text { en complément substantif }\end{array}$ & $\begin{array}{l}\text { J'en suis sûr } \\
\text { Ils en admettent l'éventualité }\end{array}$ & $\begin{array}{l}\text { Estoy seguro }\left[\boldsymbol{d e} \text { ello }{ }^{7 i}\right. \\
\text { Admiten su eventualidad }\end{array}$ \\
\hline
\end{tabular}




\begin{tabular}{|c|c|c|}
\hline $\begin{array}{l}\text { en quantitatif }(18 \%) \\
\text { en global } \\
\text { en discontinu }\end{array}$ & $\begin{array}{l}\text { Ils veulent en supprimer } \\
\text { Là où les hommes travaillaient } \\
\text { douze heures, ils n'en travailleront } \\
\text { que six }\end{array}$ & $\begin{array}{l}\text { Quieren suprimirlos } \\
\text { Lo que antes requeria que los hombres } \\
\text { trabajasen doce horas, abora sólo } \\
\text { necesita seis }\end{array}$ \\
\hline$e n$ locatif $(6 \%)$ & $\begin{array}{l}\text { Il a beaucoup de chances d'en } \\
\text { rentrer }\end{array}$ & $\begin{array}{l}\text { Tiene muchas posibilidades de volver } \\
\text { [de alli }]\end{array}$ \\
\hline $\begin{array}{l}\text { en causal }(4 \%) \\
\text { en complément d'agent } \\
\text { en culminatif }\end{array}$ & $\begin{array}{l}\text { Ils en sont bouleversés } \\
\text { Le Secret est sacré, mais il n'en est } \\
\text { pas moins un peu ridicule }\end{array}$ & $\begin{array}{l}\text { y los ha transformado } \\
\text { El Secreto es sagrado, pero no por } \\
\text { eso es un poco menos ridículo }\end{array}$ \\
\hline
\end{tabular}

\section{Y}

\begin{tabular}{c|l|l}
$y$ locatif $(60 \%)$ & Elle $\boldsymbol{y}$ est restée & Se ha quedado [alli] \\
\hline$y$ adverbial $(34 \%)$ & Il faut $\boldsymbol{y}$ mettre fin & Hace falta ponerle fin \\
\hline$y$ de construction secondaire $(3 \%)$ & & \\
$y$ complément d'adjectif & Il $\boldsymbol{y}$ est très attaché & Lo aprecia mucho \\
$y$ complément substantifiii & L'Algérie offre un bon exemple de & Argelia ofrece un buen ejemplo del \\
& cette impasse dans laquelle se & callejón sin salida en que se \\
& débattent la majorité des sociétés & encuentran casi todas las sociedades \\
& arabes. La violence $\mathbf{y}$ est née, non & árabes. En aquel país, la violencia \\
& du refus de la logique parlementaire & no surgió del rechazo a la lógica \\
& [...], mais de linterruption [...] & parlamentaria [...], sino de la \\
& des élections. & interrupción de las elecciones [...].
\end{tabular}

\section{Comparaison de la traduction de en et $y$ avec celle des autres clitiques objet}

Ensuite, le corpus a aussi permis de confronter en et $y$ et les autres clitiques français, pour examiner s'il est vrai, comme on le dit traditionnellement, que les autres clitiques objet, qui disposent bien d'un équivalent espagnol, se traduisent toujours par ces équivalents. Dans ce but j'ai étudié la traduction de 1180 de ces clitiques, répartis sur deux corpus. Les résultats démontrent que dans 20 à $30 \%$ des cas ils ne sont pas traduits par leurs équivalents directs, mais par des catégories que l'on retrouve aussi dans la traduction de en et $y$, comme, p.ex., un pronom relatif (3), un clitique sujet (c'est-à-dire, la désinence verbale espagnole (4)), un "clitique implicite" (c'est-à-dire que l'on traduit par une formulation verbale dans laquelle l'argument du clitique est vide (5), un objet direct non pronominal (6) ou un objet prépositionnel, explicite ou implicite (7).

(3) Le maire de Vienne en personne était la quatrième victime de ces lettres tueuses - il lui en a coûté trois doigts de la main gauche > La cuarta víctima de estas cartas asesinas era el mismo alcalde de Viena (que perdió tres dedos de su mano izquierda)

(4) Ca lui paraît normal > Lo percibe como normal

(5) Comme le mouvement I'avait fait en Chine > Como el movimiento [lo] bizo en China ${ }^{\text {iv }}$

(6) La France [...] ou le Royaume-Uni [...] continuent de le considérer comme moyen de symbole de puissance $>$ Francia [...] o el Reino Unido [...] continúan considerando lo nuclear como medio y simbolo de potencia.

(7) Nous ne la pleurerons pas > No lloraremos por ella 
Quelques-unes de ces catégories se retrouvent surtout dans certaines structures syntaxiques spécifiques, comme une incise ou une comparative, comme dans l'exemple $\mathrm{n}^{\circ}$ 5. Ce qui est important, c'est de noter que, sans que le traducteur y recoure, la traduction par un clitique est presque toujours possible. P.ex. les clitiques COI ne se traduisent pas en espagnol dans les cas suivants:

\begin{tabular}{l|l|l} 
ocurrence & noyau français & noyau espagnol \\
\hline 165 & dire X à Y & asegurar X [a Y] \\
\hline 119 & falloir à X & hacer falta [a X] \\
\hline 240 & naître X à Y & nacer X [a Y]
\end{tabular}

les clitiques COD ne se traduisent pas non plus dans les cas suivants:

\begin{tabular}{l|l|l}
456 & mettre $X$ en garde & advertir $[\mathrm{X}]$ \\
\hline 257 & pouvoir X & poder $[\mathrm{X}]$ \\
\hline 508 & pratiquer X & practicar $[\mathrm{X}]$ \\
\hline 406 & vouloir X & desear $[\mathrm{X}]$
\end{tabular}

Dans les cas suivants les traducteurs ont opté pour une traduction totalement différente ( $4^{\circ}$ colonne), la traduction littérale étant pourtant disponible $\left(3^{\circ}\right.$ colonne):

\begin{tabular}{l|l|l|l}
230 & entourer X & rodear X & X vivir en $\mathrm{Y}$ \\
\hline 334 & apparaître à X & parecer a X & X percibir Y \\
\hline 505 & frapper X & afectar/afligir a X & X sufrir Y
\end{tabular}

Dans aucun de ces cas il paraît y avoir des raisons syntaxiques ou discursives pour ne pas traduire par un clitique. Il y a donc plus de variation dans la traduction de ces clitiques et le cas de la traduction de en et $y$ est moins isolé qu'on ne le prétend traditionnellement. Il vaut donc mieux les traiter ensemble.

\section{Analyse linguistique de la traduction espagnole de en et $y$}

La deuxième phase de l'analyse consiste dans l'étude de la traduction de en et $y$ mêmes. Tous les 1877 cas productifs de en et $y$ ont été décrits à l'aide de 30 paramètres qui portent, e.a., sur le noyau du clitique, son référent dans le texte, son rôle sémantique vis-à-vis de ce noyau, sa catégorie sémantique, son statut valenciel, son rôle discursif dans le texte, ses caractéristiques phonétiques et prosodiques, sa distribution dans la phrase, sa distance vis-à-vis de son référent dans le texte, etc. Tous ces exemples ont alors été classifiés selon le type de en ou y et selon la manière dont ils étaient traduits. L'étape suivante consistait à essayer de découvrir dans les cas du premier corpus des relations entre ces différents types de traductions et les valeurs que donnaient les différents paramètres.

Cette confrontation a révélé que les caractéristiques phonético-prosodiques et discursives sont beaucoup plus importantes que l'on n’a jamais voulu le croire. Les clitiques se traduisent beaucoup moins par des PP parce ces deux catégories s'opposent sur les 2 plans cités: 
a) en termes discursifs, les clitiques passent de l'information donnée, tandis que les PP peuvent passer tant de l'information donnée que de l’information nouvelle, et le pouvoir référentiel de ceux-ci se situe à un niveau doublement supérieur à celui des clitiques et triplement supérieur à celui des implicites:

$$
S N(+P P)>p r . \text { tónico }>\text { pr. clítico/desinencia verbal }>0 \quad(\text { Fant }(1984: 90)
$$

ß) En termes phonético-prosodiques, les clitiques sont atones, tandis que les PP sont toniques, et s'accentuent encore plus quand ils se trouvent au début de la phrase sous une inclinaison tonale (8) et un petit peu moins quand ils se trouvent à la fin de la phrase (déclinaison tonale (9)); ils n’y a pas d'inclinaison ni de déclinaison tonale quand il se trouvent au milieu de la phrase (10) ou quand ils introduisent une relative (11).

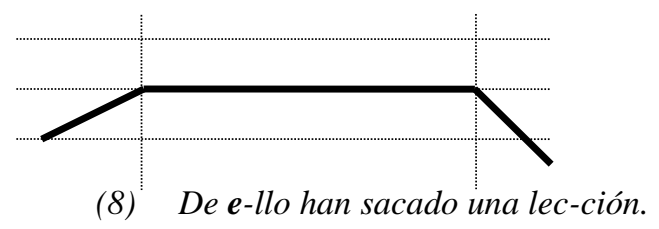

(9) Han sa-cado una lección de e-llo.

(10) Han sa-cado de ello una lec-ción.

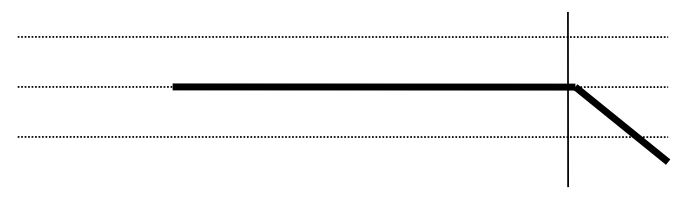

(11) ... de lo que han sacado una lec- ción.

\section{Système de traduction global}

Cette analyse a mené alors à l'élaboration d'une série d'hypothèses, qui par après ont été confrontées aux résultats du deuxième et du troisième corpus.

L' hypothèse la plus surprenante est que les traducteurs, consciemment ou inconsciemment, paraissent appliquer un système de traduction global par lequel ils essaient de trouver une traduction qui s'approche le plus possible à toutes ces caractéristiques du clitique original, de façon que, même dans un corpus de textes écrits, les règles syntaxiques (éviter une structure syntaxique marquée) et prosodiques (éviter une inclinaison ou déclinaison tonale) paraissent avoir priorité sur les règles discursives ("le thème précède le rhème", "l’information donnée précède l'information nouvelle"):

a) d'abord ils essaient de traduire par un clitique accusatif (12), datif (13) ou nominatif (14):

(12) ils $s^{\prime} \underline{\text { n }}$ souviennent $>\underline{\text { Lo }}$ recuerdan

(13) vous y ajoutez du sucre? > ile añadís azúcar? 


\section{(14) il s'en préoccupe > le preocupa}

$\beta)$ après ils recourent à un "clitique implicite" (15):

(15) ils y ajoutent du sucre $>$ añaden azúcar

$\gamma$ ) ensuite ils recourent à un implicite d'un autre type (16):

(16) il en conclut que ... > concluye que ... (vs De ello concluye que ... )

ठ) ce n'est qu'en dernier lieu qu'ils recourent à un PP, qu' ils essaient de placer d'abord au milieu (17) et, si cela n'est pas possible, à la fin (18):

(17) ils en ont tiré une bonne leçon $>$ han sacado de ello una buena lección

(18) > han sacado una buena lección de ello

ils évitent de le placer au début de la proposition, à moins que celle-ci soit une relative (dans laquelle il n'y a pas de montée tonale (19), ou que ce soit la structure syntaxique non-marquée (20):

(19) > de lo que han sacado una buena lección

(20) il en ressort que $+S u b .>$ de ello se desprende que $+S u b$. (le sujet étant une proposition, le PP s'antépose et forme avec le verbe presque une locution avec un seul accent)

Le test statistique du chi carré a démontré qu'il y a une concordance entre les fréquences des différents types de traductions dans le premier corpus et celles du deuxième corpus, et que ces types de traductions sont en plus utilisés dans les mêmes conditions (les paramètres donnant les mêmes valeurs).

Toutefois, celles du troisième corpus (le corpus TES) contiennent des déviations significatives. Celles-ci ne sont pas dues aux différents types de corpus, mais presqu'entièrement à l'usage beaucoup plus fréquent que fait un seul traducteur (le $\left.\mathrm{n}^{\circ} 19\right)$ de quelques types de traductions, en particulier des syntagmes prépositionnels au lieu de traductions implicites. Ces traductions ne sont pas vraiment incorrectes, mais elles sont moins adéquates. Il y a plusieurs indices pour cela.

En premier lieu il y a le fait qu'il ressort d'une enquête de quatre hispanophones que dans les cas où la traduction est déviante ils préfèrent presque toujours unanimement la traduction implicite à la traduction par un syntagme prépositionnel, choisie par ce traducteur.

En second lieu, j'ai calculé la récursivité des syntagmes prépositionnels dans le texte de ce traducteur, pour la comparer après avec celle d'autres textes, à savoir, celle des autres traductions et celle de textes espagnols originaux de la même série et sur le même thème que celle de la traduction déviante. Cette comparaison de la récursivité démontre que ce traducteur recourt beaucoup plus fréquemment aux syntagmes prépositionnels que ses collègues auteurs et traducteurs, ce qui revient à dire que sa traduction espagnole est moins "naturelle".

RECURSIVTTÉ DES PP

\begin{tabular}{l|l} 
& PP \\
\hline corpus TES & 17 \\
\hline les autres traductions du corpus parallèle & 14
\end{tabular}


textes journalistiques écrits en espagnol

essays écrits en espagnol

13

En troisième lieu, pas mal de verbes que le traducteur 19 construit avec un syntagme prépositionnel se trouvent aussi dans les autres traductions, mais alors sans syntagme prépositionnel explicite. P.ex. 21 vs 22:

21. Dès la conquête romaine, la vieille capitale bruissante de trafics et tourmentée périclita; il n'en resta bientôt plus pierre sur pierre derrière les enceintes écroulées, ...

22. Il donna la carcasse à un groupe d'enfants mendiants qui avalèrent ce qu'il en restait.
Tras la conquista romana, la vieja capital rumorosa de tráfico ya agitada decayó. Pronto no quedó de ella ya piedra sobre piedra detrás de las murallas derrumbadas.

Pasó la osamenta a un grupo de niños mendigos que se comieron lo que quedaba.

Et en quatrième lieu, il ressort de la comparaison des traductions par un syntagme prépositionnel avec des traductions sans celui-ci, que ces dernières sont plus appropriées à rendre toute la signification d'un clitique, y inclus ses caractéristiques discursives, qui, entre autres, sont déterminées par la phonétique et la prosodie.

\section{Stratégies de traduction spécifiques}

Cela n'empêche pas que, en dépit des écarts qu'on a pu constater dans le corpus d'un seul traducteur, l'étude de la combinais on des paramètres permette d'induire pour tous les sous-types de en et $y$ des stratégies de traduction qui tiennent compte des caractéristiques phonétiques, morphosyntaxiques, sémantiques et discursives des clitiques, sans pour autant trahir le système de l'espagnol. Ces stratégies diffèrent de manière significative des stratégies proposées dans la littérature et présentent plus de ressemblances avec la traduction des autres clitiques que l'on ne prétend généralement. Elles sont aussi accompagnées de critères de sélection, d'indices de fréquences et d'exemples (voir, en annexes, les exemples du en de construction secondaire et du en causal).

Néanmoins, les stratégies ne peuvent pas être présentées sous forme de règles catégoriques, parce que l'étude démontre aussi que la traduction de en et $y$ est basée en partie sur des paramètres partiellement subjectifs, pas entièrement objectivables ou prévisibles, comme la distance entre le clitique et son référent, l'interférence d'autres référents potentiels, la persistence du référent dans le contexte suivant et l'emploi de structures syntaxiques comme la comparaison, l'incise, etc. Ces critères peuvent réorienter le choix de la stratégie de traduction. L'absence de bons dictionnaires de valence et de régles discursives précises fait que les traducteurs hispanophones, qui ont intériorisé ces types d'information, soient mieux placés pour réaliser ces traductions que d'autres traducteurs, et surtout que les programmes informatiques, qui courent plus de risques de trahir le système espagnol, puisqu'ils ne peuvent que tenir compte de quelques valeurs moyennes. Quant à la distance entre le clitique et son référent dans le contexte antérieur, la moyenne dans le corpus est de 15 mots, avec une déviation standard de 12. En termes de propositions, la moyenne est 
de 1.4, avec une déviation standard de 1. Plus le référent est éloigné, plus explicite et précise devra être la traduction du clitique, allant de l'implicite au lexème, passant par les clitiques et les pronoms toniques. S'il y a de l'interférence, il faut expliciter la référence du clitique à l'aide d'un pronom tonique ou un lexème. Quand le référent persiste dans le contexte ultérieur, on a tendance à ne pas le répéter explicitement, pour autant que la valence verbale et la structure syntaxique le permettent et qu'il n'y ait pas d'interférence. Quant le clitique est employé de façon cataphorique, il ne se traduit pas explicitement dans la moitié des cas, surtout quand le référent est neutre et qu'il fait partie d'une incise ou une comparative.

\section{Conclusions et perspectives}

En conclusion, j'ai essayé de démontrer qu'à l'aide de l'étude quantitative et statistique combinée de corpus contextuels informatisés bilingues et monolingues, analysés à l'aide de paramètres phonétiques, prosodiques, morphosyntaxiques, sémantiques et discursifs, l'on peut arriver à induire des stratégies de traduction représentatives et polyvalentes pour des problèmes de traduction apparemment impossibles de résoudre.

Finalement, la comparaison des corpus ne permet pas de conclure que la traduction de en et $y$ diffère selon qu'il s'agisse de textes journalistiques ou littéraires. Mais pour pouvoir généraliser cela à tous les genres et régistres, il serait nécessaire d'étudier d'autres corpus. L'ensemble de textes pourrait aussi encore être diversifié diachroniquement, incluant ainsi des textes d'autres époques. Aussi serait-il utile de constituer un corpus espagnol-français, surtout pour examiner dans quel contexte apparaissent en ou $y$ quand en espagnol il n'y a rien, comme dans la traduction de ni siquiera para lamentarse par même pas pour s'en plaindre; un tel corpus permettrait aussi d'étudier dans quelle mesure les traducteurs ont tendance à traduire un syntagme prépositionnel antéposé par un syntagme prépositionnel plutôt que par un clitique. Une dernière piste intéressante serait de comparer en et $y$ avec les clitiques italiens ne et $c i$ et avec le clitique néerlandais $e r$.

\section{Bibliographie}

BADÍA MARGARIT, 1947: A. BADÍA MARGARIT, Los complementos pronominalo-adverbiales derivados de IBI e INDE en la Península ibérica, Madrid: Aguirre, 1947.

BLANCHE-BENVENISTE et alii, 1984: C. BLANCHE-BENVENISTE et alii, Pronom et syntaxe, l'approche pronominale et son application au français, Paris: Selaf, 1984.

BUYSE, 1997: K. BUYSE, La traducción española de los clíticos franceses en $e \mathrm{y}$, Leuven: thèse de doctorat de la Faculté de Lettres de la K.U. Leuven, 1997.

COSTE \& REDONDO, 1965: J. COSTE \& A. REDONDO, Syntaxe de l'espagnol moderne, Paris: Sedes, 1965.

CRIADO de VAL, 1958: M. CRIADO de VAL, Fisionomía del idioma español; sus características comparadas con las del francés, italiano, portugués, inglés y alemán, Madrid: SAETA, 1958.

DELEYE, 1988: C. DELEYE, Pour une analyse en traits du système pronominal espagnol, Leuven: mémoire de licence de la Faculté de Lettres de la K.U. Leuven, 1988. 
GARCÍA, 1975: E. GARCÍA, The role of theory in linguistic analysis. The Spanish pronoun system, Amsterdam: North-Holland, 1995.

PINCHON, 1972: J. PINCHON, Les pronoms adverbiaux EN et Y. Problèmes généraux de la représentation pronominale, Genève: Droz, 1972. 


\section{ANNEXE. STRATÉGIES DE TRADUCTION}

\section{en complément substantif}

littérature: $\quad *$ forme de base: l'implicite $*$ si /+animé/: le possessif

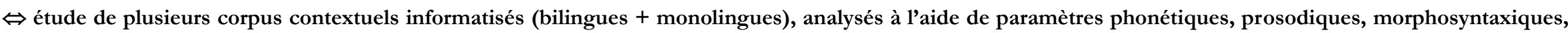
sémantiques et discursifs:

+ adjectif: + su(s) (référent $=/ \pm$ animé $/(25 \%)(1)$
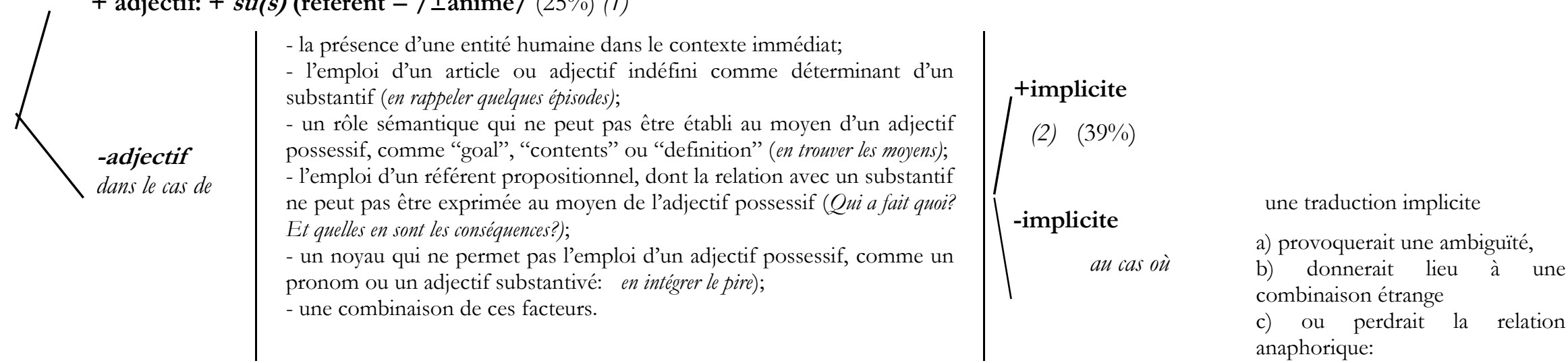

-implicite

au cas où

une traduction implicite

a) provoquerait une ambiguité, b) donnerait lieu à une combinaison étrange c) ou perdrait la relation anaphorique:

\section{$\Rightarrow$}

compl. substantif prépositionnel

$(13 \%)(3)$

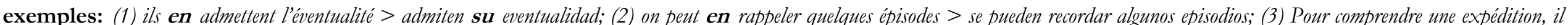
faut en donner une définition géopolitique $>$ Para entender una expedición hay que dar de ella una definición geopolitica

\section{alternatives:}

- un clitique objet $(6 \%)(4)$
- un nominatif ( \pm clitique) $(3 \%)(5)$
- un adjectif ( \pm démonstratif) $(2 \%)(6)$
(4) en apporter la confirmation > confirmarlo

(5) il en payera le prix > le costará $X$

(6) en avoir la vocation > tener la misma / esta vocación 


\section{EN CAUSAL}

\section{littérature: SPrep}

\section{$\Leftrightarrow$ étude:}

$\exists 2$ types avec \pm la même fréquence ( $=$ basse $\Rightarrow$ sans indexes de fréquence): le en complément d'agent ( $\in$ proposition passive) et le "en culminatif" (presque toujours dans une proposition active). Pour la traduction, la seule différence est que l'on dispose pour le premier type aussi du complément d'agent prépositionnel.

+implicite: la juxtaposition, coordination ou subordination des propositions suffit pour maintenir la référence (1)

-implicite: ne suffit pas $\Rightarrow$

$$
\begin{aligned}
& \text { si "en V pas moins" } \Rightarrow \text { connecteur concessif (7) ou PP, introduit par por et antéposé au verbe (5) } \\
& \text { autres } \Rightarrow \mid \begin{array}{l}
\text { ou un connecteur comme pero, así, de esa forma, de modo que ... (2+3) } \\
\text { ou un nominatif (+/-subordonnée relative) (4) } \\
\text { ou un } \mathbf{P P}, \text { introduit par por et antéposé au verbe (5) }
\end{array}
\end{aligned}
$$

alternative, déconseillée par les informateurs: complément d'agent prépositionnel (6)

\section{exemples:}

(1) Une jonquille toute dorée effleure, sans en paraître effarouchée, une chenille de tank > Un junco de color de oro, sin parecer intimidado, brota entre la cadena de un tanque

(2) La menace n'en est pas éloignée pour autant > Pero esta amenaza sigue existiendo

(3) Il n'en finit pas de féliciter l'élève modèle $>$... de modo que no para de felicitar al alumno ejemplar

(4) Ils en sont bouleversés $>$ y los ha transformado / que los ha transformado

(5) Le Secret est sacré, mais il n'en est pas moins un peu ridicule $>$ El Secreto es sagrado, pero no por eso es un poco menos ridiculo

(6) La vie du monastère n'en est pas dérangée > La vida del monasterio no es alterada por ello

$$
\text { 7) }
$$

TES 12.159. Elle [l'accumulation de prodiges, k.b.] révèle chez Benoît deux dons essentiels: l'un, de pénétration des esprits et des âmes qui, divinatoire dans ses effets, n'en est pas moins d'ordre naturel.
[La colección, k.b.] revela en Benito dos dones esenciales: uno, de penetración de los espíritus y de las almas que, aunque adivinatorio en sus efectos, no es menos de orden natural. 


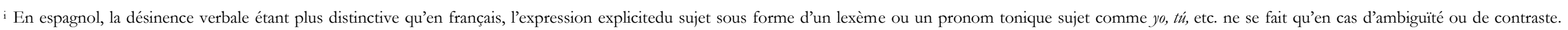
C’est pourquoi la désinence verbale est parfois appelée clitique sujet (García (1975: 73); Buyse (1997: 74-79)).

‘ $\phi$ ’ implicite.

ii []$=$ implicite.

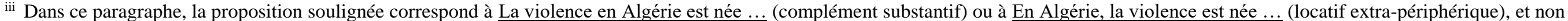
pas à La violence est née en Algérie. Pour une discussion plus détaillée, voir Buyse (1997: 271-272).

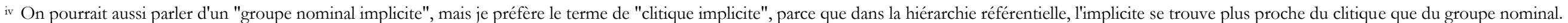

